\title{
KY19382, a novel activator of Wnt/ $\beta$-catenin signaling, promotes hair re-growth and hair follicle neogenesis
}

\author{
YEONG CHAN RYU ${ }^{1}$, Dong-Hwan Lee ${ }^{1}$, Jiyong Shim ${ }^{1}$, Jiyeon Park ${ }^{1}$, You-rin Kim ${ }^{1}$, Sehee \\ $\mathrm{Choi}^{1}$, Soon Sun Bak ${ }^{2}$, Young Kwan Sung ${ }^{2}$, Soung-Hoon Lee ${ }^{1}$, and Kang-Yell Choi ${ }^{1}$
}

${ }^{1}$ Yonsei University

${ }^{2}$ Kyungpook National University

June 15,2020

\begin{abstract}
Background and Purpose: The promotion of hair regeneration and growth heavily depends on the activation of Wnt/ $\beta$ catenin signaling in the hair follicle including dermal papilla (DP). KY19382, one of the newly synthesized analogs of indirubin-3'monoxime ( $\mathrm{I} 3 \mathrm{O})$ was identified as a Wnt/ $\beta$-catenin signaling activator via inhibition of the interaction between CXXC-type zinc finger protein 5 (CXXC5) and Dishevelled (Dvl) interaction. Given the close relationship between the Wnt/ $\beta$-catenin signaling and hair regeneration, we investigated the effect of KY19382 on hair re-growth and hair follicle neogenesis. Experimental Approach: In vitro hair induction effects of KY19382 was performed in human dermal papilla cells. The hair elongation effects of KY19382 was confirmed through the vibrissa culture system. In vivo hair regeneration abilities of KY19382 was identified in three models: hair regrowth, wound-induced hair follicle neogenesis (WIHN), and hair patch assays using C57BL/6 mice. The hair regeneration abilities were analyzed by immunoblotting, alkaline phosphatase (ALP) and immunohistochemical staining. Key results: KY19382 activated Wnt/B-catenin signaling and elevated the expression of ALP and proliferation marker PCNA in DP cells. KY19382 also increased hair length in ex vivo cultured mouse vibrissa follicles and induced hair regrowth in mice. Moreover, KY19382 significantly promoted the generation of de novo hair follicles as shown by WIHN and hair patch assays. Conclusion and Implications: These results indicate that KY19382 is a potential therapeutic drug that exhibits effective hair regeneration ability via activation of the Wnt/ $\beta$-catenin signaling for alopecia treatments.
\end{abstract}

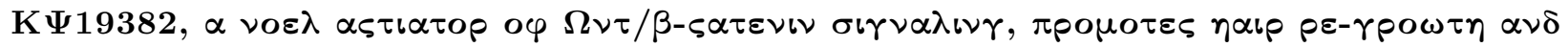

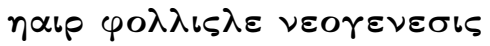

Yeong Chan Ryu ${ }^{1}$, Dong-Hwan Lee ${ }^{1}$, Jiyong Shim ${ }^{1}$, Jiyeon Park ${ }^{1}$, You-rin Kim ${ }^{1}$, Sehee Choi ${ }^{1}$, Soon Sun $\mathrm{Bak}^{3}$, Young Kwan Sung ${ }^{3}$, Soung-Hoon Lee ${ }^{2,}$, and Kang-Yell Choi ${ }^{1,2,}{ }^{*}$

${ }^{1}$ Department of Biotechnology, College of Life Science and Biotechnology, Yonsei University, Seoul, Korea

${ }^{2}$ CK Biotechnology Inc, Engineering Research Park, 50 Yonsei Ro, Seodaemun-Gu, Seoul 03722, Korea

${ }^{3}$ Department of Immunology, School of Medicine, Kyungpook National University, Daegu 41944, Korea.

*Address correspondence to:

Kang-Yell Choi or Soung-Hoon Lee, College of Life Science and Biotechnology, Yonsei University

134 Shinchon-Dong, Seodaemun-Gu, Seoul 03722, Korea

Tel: +82-2-2123-7438; Fax: +82-2-2123-8284

E-mail: kychoi@yonsei.ac.kr; sexyondal@gmail.com 
Background and Purpose: The promotion of hair regeneration and growth heavily depends on the activation of Wnt/ $\beta$-catenin signaling in the hair follicle including dermal papilla (DP). KY19382, one of the newly synthesized analogs of indirubin-3'-monoxime (I3O) was identified as a Wnt/ $\beta$-catenin signaling activator via inhibition of the interaction between CXXC-type zinc finger protein 5 (CXXC5) and Dishevelled (Dvl) interaction. Given the close relationship between the Wnt/ $\beta$-catenin signaling and hair regeneration, we investigated the effect of KY19382 on hair re-growth and hair follicle neogenesis.

Experimental Approach: In vitro hair induction effects of KY19382 was performed in human dermal papilla cells. The hair elongation effects of KY19382 was confirmed through the vibrissa culture system. In vivo hair regeneration abilities of KY19382 was identified in three models: hair regrowth, wound-induced hair follicle neogenesis (WIHN), and hair patch assays using C57BL/6 mice. The hair regeneration abilities were analyzed by immunoblotting, alkaline phosphatase (ALP) and immunohistochemical staining.

Key results : KY19382 activated Wnt/ $\beta$-catenin signaling and elevated the expression of ALP and proliferation marker PCNA in DP cells. KY19382 also increased hair length in ex vivo cultured mouse vibrissa follicles and induced hair regrowth in mice. Moreover, KY19382 significantly promoted the generation of de novo hair follicles as shown by WIHN and hair patch assays.

Conclusion and Implications: These results indicate that KY19382 is a potential therapeutic drug that exhibits effective hair regeneration ability via activation of the Wnt/ $\beta$-catenin signaling for alopecia treatments.

Key words: Dermal papilla cells, Wnt/ $\beta$-catenin signaling, GSK-3 $\beta$, CXXC5, neogenesis

\section{What is already known}

- Wnt/ $\beta$-catenin signaling plays important role in hair regrowth and regeneration.

- CXXC5 inhibits hair regeneration by suppressing the Wnt/ $\beta$-catenin signaling pathway.

\section{What this study adds}

- Newly discovered small molecule promotes hair regeneration by simultaneously inhibiting GSK-3 $\beta$ and blocking CXXC5-Dvl interaction.

- This molecule induces HFSC activation and hair induction marker, unlike commercial hair growth promoting agents.

\section{What is the clinical significance}

- Drugs enhancing WIHN are commercially unavailable but need to be developed for alopecia treatment.

- Dual targeting of GSK-3 $\beta$ and CXXC5-Dvl interaction could be a new treatment for baldness.

\section{INTRODUCTION}

Recent reports show a significant increase in the number of people with alopecia (Jang et al., 2013). In the skin of alopecia patients, many hair follicles overstay in their resting stage in which dermal papilla (DP) cells and keratinocytes, the two key players of hair cycle regulation, are inactive (Alonso \& Fuchs, 2006; Botchkarev \& Kishimoto, 2003; Sennett \& Rendl, 2012). Inactivation of these cells is caused by various factors, and the inactivation leads to miniaturized hair follicles, resulting hair loss (Kligman, 1959; Price, 1999). Even though researchers have focused on developing drugs to cure alopecia, only two drugs, minoxidil (MNX) and finasteride, have been approved by the US Food and Drug Administration for clinical treatment of androgenic alopecia (Libecco \& Bergfeld, 2004; Linas \& Nies, 1981; Price, 1999). Although both drugs are effective in promoting hair growth, neither can initiate hair follicle neogenesis effectively due to complicated process of the hair regeneration (Paus, 2006).

Wnt/ $\beta$-catenin signaling pathway plays an important role in hair morphogenesis, growth initiation, and regeneration (Andl, Reddy, Gaddapara, \& Millar, 2002; Huelsken, Vogel, Erdmann, Cotsarelis, \& Birchmeier, 2001; Iida, Ihara, \& Matsuzaki, 2007; Ito et al., 2007; Kishimoto, Burgeson, \& Morgan, 2000). The activated Wnt/ $\beta$-catenin signaling pathway initiates embryonic hair formation in the epidermis and promotes the 
formation of dermal condensates (Andl et al., 2002; Huelsken et al., 2001). In addition, DP cells require activation of this pathway to initiate hair-inducing activity that prolongs the anagen phase (Kishimoto et al., 2000). The hair-inducing ability of DP cells can be confirmed by analyzing the expression of alkaline phosphatase (ALP) (Iida et al., 2007; S. H. Lee et al., 2012). Recent studies showed that the activation of Wnt/ $\beta$-catenin signaling can recover ALP expression in long-term cultured primary DP cells (Yamauchi \& Kurosaka, 2009).

Hair growth promoting drugs targeting Wnt/ $\beta$-catenin signaling are not currently available. However small molecules are needed that, unlike MNX, can activate this pathway to enhance hair follicle neogenesis (S. H. Lee et al., 2017).

KY19382 is, one of the newly synthesized analogs of indirubin-3'-monoxime (I3O), a glycogen synthase kinase$3 \beta$ (GSK-3 $\beta$ ) inhibitor, and has a significant ability to activate the Wnt/ $\beta$-catenin signaling. Indirubin is an active ingredient of the indigo plant, Danggui Longhui Wan, used as a traditional anti-leukemia medicine (Xiao, Hao, Liu, \& Qian, 2002). I3O is known as a Wnt/ $\beta$-catenin activator that stabilizes $\beta$-catenin via inhibition of GSK-3 $\beta$, and has been shown to accelerate bone growth and inhibit adipogenesis (O. M. Choi et al., 2014; Zahoor, Cha, \& Choi, 2014; Zahoor, Cha, Min do, \& Choi, 2014). Furthermore, we recently found that KY19382 elongated tibial length by inactivating GSK-3 $\beta$ and blocking the binding of CXXC-type zinc finger protein 5 (CXXC5) and Dishevelled (Dvl) (S. Choi et al., 2019). Moreover, PTD-DBM, a peptide that interferes with CXXC5-Dvl interaction via binding to the PDZ domain of Dvl, stimulated wound-induced hair follicle neogenesis (WIHN) and hair regrowth (Lee et al., 2017). However, this peptide is limited for routine application due to its cost and stability.

In this study, we selected KY19382 as an optimal compound that can stimulate hair growth. KY19382 activated the Wnt/ $\beta$-catenin signaling higher than $\mathrm{I} 3 \mathrm{O}$ and showed low cytotoxicity in human DP cells. The KY19382 elongated vibrissa hair shaft and mouse dorsal hair. Moreover, KY19382 significantly induced the hair follicle neogenesis as shown in hairless mice injected with dermal cells and keratinocytes and wounded mice treated with KY19382. Overall, KY19382 is an effective Wnt/ß-catenin signaling activator that can be used for treatment of alopecia with high efficacy and safety.

\section{METHODS}

\subsection{Cell culture and reagents}

Primary human DP cells from passages 2-7 were used in this study (Kwack et al., 2013). The cells were cultured in Dulbecco's low glucose modified Eagle's medium (Hyclone, Pittsburgh, PA) supplemented with $10 \%$ fetal bovine serum (Gibco, Gaithersburg, MD), $1 \%$ antibiotic-antimycotic (Gibco), $1 \mathrm{ng} / \mathrm{ml} \mathrm{bFGF}$ (Peprotech, Princeton, NJ), and $5 \mu \mathrm{g} / \mathrm{ml}$ insulin (Gibco) at 37 in a humidified atmosphere of $5 \% \mathrm{CO}_{2}$. A rat vibrissa immortalized DP cell line was donated by the Skin Research Institute of the Amore Pacific Corporation R\&D Center (Yongin, Korea). HEK293T cells containing a chromosomally integrated TCF reporter (TOPflash) were cultured in Dulbecco's Modified Eagle Medium (DMEM) (Gibco) containing 10\% (v/v) fetal bovine serum (Gibco) and $100 \mathrm{U} / \mathrm{ml}$ penicillin/streptomycin (Gibco) at $37^{\circ} \mathrm{C}$ in a humidified atmosphere of $5 \% \mathrm{CO}_{2}$.

\section{2 in vivo hair growth test}

Six-week-old C57BL/6N male mice were purchased from Koatech Co. (Gyeonggido, Korea) and acclimated for 1 week. The hairs on the backs of 7 -week-old mice, whose hair follicles were in telogen phase, were shaved with a hair clipper. For each of the mice, $300 \mu \mathrm{l}$ of each drug was applied topically daily at an appropriate concentration (as described in figure legends) for up to 14 or 28 days. KY19382 was dissolved in a PEG400 and $\beta$-cyclodextrin (vehicle 1), and minoxidil was dissolved in $50 \%$ (vol/vol) ethanol, $30 \%$ water, and $20 \%$ propylene glycol (vehicle 2).

\subsection{Wound-induced hair follicle neogenesis assay}

Three-week-old C57BL/6N male mice were allowed to adapt to their new environment for 3 days $.1 \mathrm{~cm}^{2}$ 
full-thickness wounds were generated on the backs of the mice. $20 \mu \mathrm{l}$ of each drug was applied to the wounds daily for up to 14, 25, or 40 days. All animal experiments were approved by the Institutional Animal Care and Use Committee of Yonsei University.

\subsection{Luciferase reporter assay}

HEK293T cells were treated with KY19382 in 24-well plates for 24 hours. The cells were washed with cold phosphate buffered saline (PBS) and lysed in $55 \mu \mathrm{l}$ of $1 \mathrm{x}$ lysis buffer (Promega, Madison, WI). The cell lysates were centrifuged at $15,920 \mathrm{x}$ g at $4^{\circ} \mathrm{C}$ for 15 minutes.

Thirty microliters of each supernatant was transferred to 96-well plates and $15 \mu \mathrm{l}$ luciferin was added. The luciferase activity was measured at $485 \mathrm{~nm}$ using a FLUOstar OPTIMA luminometer (BMG Labtech, Offenburg, Germany).

\subsection{Immunoblotting}

Human or rat DP cells were incubated with KY19382 for 48 hours. The cells were washed twice with cold PBS and lysed in RIPA buffer $(150 \mathrm{mM} \mathrm{NaCl} ; 10 \mathrm{mM}$ Tris, pH 7.2; $0.1 \%$ SDS; $1 \%$ Triton X-100; $1 \%$ sodium deoxycholate; $5 \mathrm{mM}$ EDTA). Cell lysates were centrifuged at $15,920 \mathrm{x}$ g at $4^{\circ} \mathrm{C}$ for 30 minutes. The protein was separated on 8-10\% SDS PAGE gels and transferred onto PROTRAN nitrocellulose membranes (Schleicher and Schuell Co., Keene, NH). After blocking with $5 \%$ skim milk for 1 hour at room temperature, each membrane was blotted with the following primary antibodies: anti- $\beta$-catenin $(1: 1000$, Santa Cruz Technology, Dallas, TX or 1:1000, Cell Signaling, Beverly, MA), anti- $\alpha$-tubulin (1:4000, Cell Signaling or 1:2000, Oncogene Research Products, Cambridge, MA), anti-p-GSK-3 $\beta$ (S9) (1:1000, Cell Signaling), antiPCNA (1:500, Santa Cruz Technology or 1:1000, Cell Signaling), anti-FGF9 (1:1000, Abcam, Cambridge, MA) and anti-cytokeratin 17 (1:1000, Abcam) at $4^{\circ} \mathrm{C}$ overnight. Each membrane was blotted with horseradish peroxidase-conjugated anti-mouse (1:3000, Cell Signaling) or anti-rabbit (1:3000, Cell Signaling) IgG secondary antibody. The blots were visualized using enhanced chemiluminescence (Amersham Bioscience, Buckinghamshire, UK) and a luminescent image analyzer (LAS-4000; Fujifilm, Tokyo, Japan).

\subsection{Cell viability assay}

Human DP or rat DP cells were seeded in 24-well plates and treated with KY19382 for 48 hours. After adding $50 \mu \mathrm{l}$ cell titer reagent to each well, the plates were incubated for 10 minutes at room temperature. The absorbance was measured using the FLUOstar OPTIMA luminometer.

\subsection{Immunocytochemistry}

Human or rat DP cells were seeded in 12-well plate on cover slips. The cells were incubated with KY19382 for $48 \mathrm{~h}$. Cultured cells were washed twice with cold PBS and were fixed in $4 \%$ paraformaldehyde or $10 \%$ formalin for $15 \mathrm{~min}$ at room temperature, and then were washed with PBS and permeabilized with $0.2 \%$ Triton X-100 for 15 min. After blocking with 5\% BSA in PBS for $30 \mathrm{~min}$ at room temperature, the cells were blotted with primary antibody: $\beta$-catenin antibody (1:100, BD Transduction Laboratory, Lexington, $\mathrm{KY}$ or $1: 50, \mathrm{Abcam})$ overnight at $4^{\circ} \mathrm{C}$. After washing with PBS, the cells were blotted with Alexa Fluor 488-conjugated goat anti-mouse antibody or Alexa Fluor 555-conjugated goat anti-rabbit antibody (1:300, Molecular Probes, Leiden, The Netherlands) for $1 \mathrm{~h}$ at room temperature and counterstained with 4',6diamidino-2-phenylindole (DAPI; 1:5000, Boehringer Mannheim, Mannheim, Germany) for 10 min at room temperature. Images were taken using a LSM510 confocal microscope (Carl Zeiss Inc., Thornwood, NY). The fluorescence intensity was quantified using NIS-Elements AR 3.2 software (Nikon).

\subsection{Alkaline phosphatase staining}

For ALP staining in cells, human or rat DP cells were seeded in 12-well plate on coverslips. Cells were incubated with KY19382 for 48 hours and washed twice with cold PBS and were fixed in formalin for 15 minutes at room temperature. Then cells were incubated with nitro-blue tetrazolium and 5-bromo-4-chloro3'-indolyphosphate solution (NBT/BCIP solution, Sigma Aldrich). The reaction was stopped by washing with PBS. Dark blue staining was a positive signal for ALP. 
For ALP staining of tissues, 20- $\mu \mathrm{m}$ cryosections were dried for 4 hours, then fixed in $4 \%$ paraformaldehyde for 5 minutes. After being washed with PBS, the sections were incubated in NBT/BCIP solution (NBT/BCIP tablets, Roche Diagnostics, Rotkreuz, Switzerland) for 30 minutes. The slides were counterstained with nuclear fast red solution (Sigma Aldrich) for 30 seconds, washed in distilled water, and then dried for 2 hours. The slides were incubated in $100 \%$ xylene for 30 seconds, then mounted using Permount.

For whole mount ALP staining, the wounded skin tissues were incubated $5 \mathrm{mM}$ EDTA in PBS at $37{ }^{\circ} \mathrm{C}$ for 6 hours. The dermis was separated under the microscope (Eclipse TE2000-U; Nikon). The dermis was fixed in $4 \%$ paraformaldehyde for 10 minutes, washed with PBS and then incubated in NBT/BCIP solution (NBT/BCIP tablets, Roche Diagnostics) for 10 minutes.

\subsection{ALP activity assay}

Human or rat DP cells were seeded in 24-well plates and incubated with KY19382 for 48 hours. Next, the cells were washed twice with cold PBS and lysed with $55 \mu \mathrm{l} 1 \mathrm{x}$ reporter lysis buffer (Promega) per well. Cell lysates were centrifuged at $10,000 \mathrm{x} g$ at $4^{\circ} \mathrm{C}$ for 30 minutes. Thirty microliters of each supernatant was incubated with $30 \mu \mathrm{l}$ of p-nitrophenyl phosphate (pNPP) liquid substrate (Sigma Aldrich) for 1 hour. ALP activity was measured at $405 \mathrm{~nm}$ using the FLUOstar OPTIMA luminometer and normalized by the protein concentration from the Bradford assay (Bio-Rad Laboratories, Hercules, CA).

\section{siRNA preparation and transfection}

The cells were transfected with siRNA or the negative control (Bioneer, Daejeon, Korea) using Lipofectamine Plus (Invitrogen, Carlsbad, CA) in serum-free Opti-MEM (Gibco) according to the manufacturer's instructions at a final concentration of $100 \mathrm{nM}$. The siRNA sequences targeting $\beta$-catenin were 5'-GAAACGGCTTTCAGTTGAG-3' and 5'-AAACTACTGTGGACCACAAGC-3' (Bioneer). At 12 hours after transfection with $\beta$-catenin siRNA, cells were treated with KY19382 for 48 hours. ALP assay and immunoblotting were performed to examine changes in ALP activity and to confirm the transfection efficiency of $\beta$-catenin siRNA.

\subsection{Ex vivo vibrissa follicle culture}

Mouse vibrissa follicles were isolated from a C57BL/6 mouse. After euthanizing the mouse, anagen follicles were isolated for organ culture under a stereomicroscope (Nikon). The isolated follicles were placed in 500 $\mu \mathrm{l}$ DMEM supplemented with $100 \mathrm{U} / \mathrm{ml}$ penicillin/streptomycin (Gibco) and $12.5 \mu \mathrm{g} / \mathrm{ml}$ gentamicin (Gibco) in 24-well plates. The vibrissa follicles were treated with DMSO, KY19382 $(5 \mu \mathrm{M})$ or MNX $(100 \mu \mathrm{M})$. The culture medium was changed every 2 days.

Rat vibrissa follicles were isolated from a 21-day-old male Wistar rat (Philpott, Green, \& Kealey, 1992). After euthanizing the rat, anagen follicles were isolated under a stereomicroscope (Nikon). The culture medium was changed every 4 days.

\subsection{X-gal staining}

Tissues were fixed in $0.4 \%$ paraformaldehyde for 3 hours and $0.2 \%$ glutaraldehyde for 15 minutes, then washed with PBS. The tissues were incubated in X-gal solution at $37^{\circ} \mathrm{C}$ overnight.

\subsection{Hematoxylin and eosin staining}

Skin tissues were fixed in $4 \%$ paraformaldehyde or $10 \%$ formalin overnight at $4^{\circ} \mathrm{C}$. The tissues were dehydrated, paraffinized, embedded in paraffin and sliced into $4 \mu \mathrm{m}$ thickness. The paraffin sections were rehydrated through xylene and graded ethanol series. The slides were incubated in hematoxylin for 5 minutes and eosin for 1 minute.

\subsection{Immunohistochemistry (IHC)}

Paraffin sections were deparaffinized and rehydrated. For antigen retrieval, the slides were autoclaved or microwaved for 15 minutes in $10 \mathrm{mM}$ sodium citrate buffer. The samples were pre-incubated in PBS and 
then blocked with 5\% BSA in PBS for 30 minutes at room temperature. The samples were incubated overnight at $4^{\circ} \mathrm{C}$ with the following primary antibodies: anti- $\beta$-catenin (1:200, BD Transduction or 1:100, Abcam), anti-K15 (1:200, Abcam), anti-PCNA (1:500, Santa Cruz Technology), anti-Ki67 (1:500, Abcam), anti-Fgf9 (1:500, Abcam) and anti-Cytokeratin 17 (1:500, Abcam). After washing with PBS, the slides were incubated with anti-mouse Alexa Fluor 488 or anti-rabbit Alexa Fluor 555 conjugated secondary antibody (1:500, Molecular Probes) for 1 hour at room temperature and counterstained with DAPI (1:3000, Boehringer Mannheim) for 10 minutes. The images were taken using a LSM510 confocal microscope (Carl Zeiss).

\subsection{Hair patch assay}

Epidermal and dermal cells were isolated from neonatal mice. The skin of neonatal mice was peeled and digested with $0.25 \% \beta$-trypsin to separate the epidermis and dermis, and the separated dermal cells were seeded in $100 \mathrm{~mm}$ culture dish and incubated with $5 \mu \mathrm{M}$ KY19382 or DMSO for 72 hours. $1 \times 10^{6}$ epidermal and $1.5 \times 10^{6}$ dermal cells were injected subcutaneously into hairless mice.

\subsection{Statistical analysis}

Data are presented as the means \pm SE. Statistical analyses were performed using an unpaired two-tailed Student's t-test. Statistical significance is indicated in the figures as follows: ${ }^{*} \mathrm{P}<0.05,{ }^{* *} \mathrm{P}<0.01,{ }^{* * *} \mathrm{P}$ $<0.001$

\section{RESULTS}

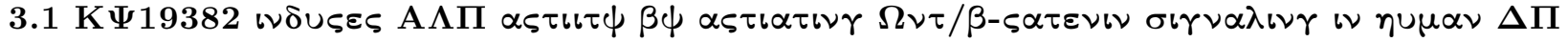 $\varsigma \varepsilon \lambda \lambda \varsigma$}

KY19382, a newly synthesized small molecule derived from I3O, was screened by its capability to interfere with the CXXC5-Dvl protein-protein interaction, followed by activation of Wnt/ $\beta$-catenin signaling (S. Choi et al., 2019). KY19382 showed significantly higher Wnt activity than I3O in a dose-dependent manner in HEK293T cells (Figure 1a). To determine the highest concentration of KY19382 without toxic effects in rat DP cells, cell viability was measured in multiple doses. Cell viability was not significantly affected at 1 and $5 \mu \mathrm{M}$ KY19382 but decreased at $10 \mu \mathrm{M}$ KY19382 for rat DP cells (Supplementary figure 1a). In addition, 1 and $5 \mu \mathrm{M}$ KY19382 did not show any toxicity in human DP cells (Figure 1b). Thus, 1 and $5 \mu \mathrm{M} \mathrm{KY19382}$ was used for subsequent in vitro studies.

To determine whether KY19382 activates Wnt/ $\beta$-catenin signaling via GSK-3 $\beta$ inhibition, we examined the levels of $\beta$-catenin and p-GSK-3 $\beta$ (S9), an inactive form of GSK-3 $\beta$, in DP cells. When treated with KY19382, the expression levels of $\beta$-catenin, p-GSK-3 $\beta$ (S9), and proliferation marker, PCNA were increased in DP cells (Figure 1c and supplementary figure $1 \mathrm{~b}$ ). The upregulation of Wnt/ $\beta$-catenin signaling was further confirmed by cytochemical anaylsis of DP cells treated with KY19382 (Figure 1d and supplementary figure 1c). To assess the effect of KY19382 on ALP activity induction, we performed ALP staining and ALP activity assays. KY19382 treatment resulted in higher ALP staining intensity and activity compared to those in the non-treated control (Figure 1e, 1f and supplementary figure 1d, 1e).

The effect of Wnt/ $\beta$-catenin signaling on KY19382-induced ALP activity was confirmed by using human $\beta$-catenin siRNA transfection on human DP cells. The knock-down effect of $\beta$-catenin siRNA transfection was verified using immunoblotting analysis (Supplementary figure 2a). KY19382-induced ALP expression was abolished by transfection with $\beta$-catenin siRNA in human DP cells, however this inhibitory effect was not shown in cells transfected with a control siRNA (Figure $1 \mathrm{~g}$ ). The quantification of ALP activity was also confirmed that KY19382 induces ALP activity via the Wnt/ $\beta$-catenin signaling (Figure 1h).

Overall, these data showed that KY19382 significantly activated Wnt/ $\beta$-catenin signaling in both human and rat DP cells without cytotoxicity. In addition, KY19382 treatment elevated ALP activity by activating Wnt/ $\beta$-catenin signaling.

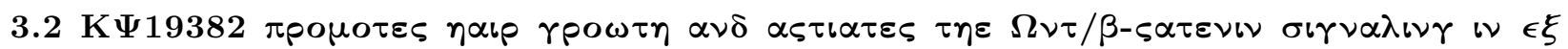

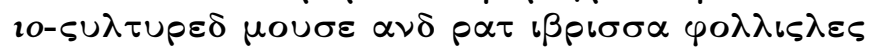


To determine whether KY19382 promotes hair follicle growth, we treated mouse and rat vibrissa follicles with KY19382 ex vivo. KY19382 treatment on mouse vibrissa follicles for 6 days and rat vibrissa follicles for 12 days significantly promoted vibrissa follicle elongation. (Figure 2a and supplementary figure 3a).

To confirm the effect of KY19382 on the activation of Wnt/ $\beta$-catenin signaling in mouse vibrissa follicles, we performed immunohistochemical (IHC) analyses. An increase in $\beta$-catenin and proliferation marker expression was observed in mouse and rat vibrissa follicles (Figure $2 \mathrm{~b}$ and supplementary figure 3b, 3c). Moreover, increased ALP expression was detected in cultured mouse vibrissa follicles treated with KY19382, demonstrating the hair-inducing effect of KY19382 (Figure 2c). Correspondingly, LacZ expression was highly increased at the base of mouse vibrissa follicles from Axin $2^{\text {lacZ/+ }}$ mice after KY19382 treatment, showing its strong effect on Wnt/ $\beta$-catenin signaling activation (Figure $2 \mathrm{~d}$ ).

In summary, $\beta$-catenin, ALP, and Axin2-LacZ expression levels were concomitantly increased at the hair bulb when treated with KY19382, suggesting that KY19382 accelerated vibrissa follicle growth and activated Wnt/ $\beta$-catenin signaling ex vivo .

\subsection{KY19382 promotes hair regrowth in mice}

To investigate the effect of KY19382 on mouse hair regrowth, KY19382 or vehicle was topically applied to the shaved dorsal skin of 7-week-old mice daily for 14 or 28 days. MNX was used as the positive control (S. H. Lee et al., 2012). After 28 days, KY19382 promoted hair regrowth more efficiently than MNX (Figure 3a). Hematoxylin and eosin $(\mathrm{H} \& \mathrm{E})$ staining showed that hair follicles in the control group were still in the telogen phase, but hair follicles of skin treated with KY19382 or MNX for 28 days entered anagen phase (Figure 3b). To confirm KY19382-induced changes in proliferation or Wnt/ $\beta$-catenin signaling in the bulge, IHC analyses were performed on skin tissues treated for 14 days. Proliferation markers, ki67 and PCNA, were specifically increased in keratin 15-positive bulge stem cells of the KY19382-treated group (Figure 3c and supplementary figure 4a). $\beta$-Catenin was also increased only in the KY19382-treated group. Similarly, western blot analyses showed that the levels of $\beta$-catenin and PCNA were significantly increased in the KY19382-treated group (Figure 3d). Moreover, ALP, a critical marker for hair induction, was highly expressed in the DP cells of skins treated with KY19382 for 14 days (Figure 3e). Collectively, these data suggested that KY19382 promoted hair regrowth and increased markers for hair growth promotion, such as $\beta$-catenin, PCNA and ALP more efficiently than MNX.

\subsection{KY19382 enhances wound-induced hair follicle neogenesis}

Considering that KY19382 stimulated hair growth in in vitro, ex vivo and in vivo systems via activation of Wnt/ $\beta$-catenin signaling, the effect of KY19382 on WIHN was tested in mice.

To confirm this, we cut $1 \mathrm{~cm}^{2}$ full thickness wounds in 3-4-week-old mice and applied the drugs daily for 14, 25, and 40 days after wounding. The KY19382-treated group increased number of newly formed follicles compared to those in the vehicle treated group as confirmed by whole mount ALP staining (Figure 4a). In addition, H\&E staining showed that KY19382 induced formation of neogenic follicles 14 days post-wounding (Figure $4 \mathrm{~b}$ ). IHC analyses showed that keratin 17, a marker for intermediate filament keratin protein (Ito et al., 2007), was specifically increased in neogenic follicles of the KY19382-treated group (Figure 4c). Fgf9 involved in WIHN in wound fibroblasts (S. H. Lee et al., 2017) was also increased in the dermis of wounds treated with KY19382 (Figure 4c). $\beta$-Catenin and proliferation markers, ki67 and PCNA were increased in the KY19382-treated group, especially in the neogenic follicles of wounds (Figure 4c and supplementary figure 5a). Furthermore, western blot analyses confirmed that the markers associated with hair follicle neogenesis were increased in wounded skin treated with KY19382, but not MNX (Figure 4d). The newly formed white hairs at the wound sites were found only in the KY19382-treated group (Figure 4e). Taken together, KY19382 markedly induced WIHN and WIHN-related markers by significantly activating Wnt/ $\beta$-catenin pathway.

\subsection{KY19382 promotes hair follicle neogenesis in patch assays}

To investigate the therapeutic effect of KY19382, we utilized a hair patch assay system. Mouse dermal cells 
were treated with $5 \mu \mathrm{M}$ KY19382 for 72 hours prior to transplantation, then mixed with epithelial cells for injection into hairless mice.

After 14 days post-injection, reconstituted hair follicles were observed on the skin of hairless mice where cells were injected (Figure 5a). The number and density of neogenic hair follicles generated in the tissue injected with the KY19382-treated cells were significantly higher than those injected with the non-treated control cells (Figure 5b). Moreover, the expression levels of $\beta$-catenin and Ki67 were greatly increased in neo-generated hair follicles induced by KY19382 as demonstrated by IHC analysis (Figure 5c).

\section{DISSCUSSION}

Currently available drugs for treating alopecia are limited due to their inability to regenerate hair follicles. MNX and finasteride can promote hair growth when the hair follicle is present, but they are not effective in patients with severe alopecia (Libecco \& Bergfeld, 2004; Messenger \& Rundegren, 2004; Price, 1999; Rossi et al., 2016). Existing drugs that control the proliferation of hair cells can be difficult for treating patients with miniaturized or absent hair follicles (Han et al., 2004). Therefore, we aimed to develop a drug that is effective in promoting hair regrowth and hair follicle neogenesis by inducing markers for hair induction such as ALP and activating hair follicle stem cells via Wnt/ $\beta$-catenin pathway.

Hair follicle neogenesis could be an important strategy for alopecia treatment (Ito et al., 2007). Previous studies have shown that hair follicle neogenesis highly depends on the activation of Wnt/ $\mathrm{B}$-catenin signaling in DP cells and keratinocytes, along with activation of hair follicle stem cells (Enshell-Seijffers, Lindon, Kashiwagi, \& Morgan, 2010; Huelsken et al., 2001; Ito et al., 2007; Waters, Richardson, \& Jahoda, 2007). Wnt/ $\beta$-catenin signaling plays essential roles in maintaining the hair-inducing ability of DP cells and promoting hair follicles to the anagen phase (Andl et al., 2002; Kishimoto et al., 2000; Sick, Reinker, Timmer, \& Schlake, 2006). In addition, previous literature has suggested that Wnt/ $\beta$-catenin signaling activators, such as valproic acid (S. H. Lee et al., 2012), Aconiti ciliare tuberextract (Park et al., 2012), and Malva verticillata seed extract (E. Y. Lee et al., 2016), are potential candidates for alternative hair growth treatments as they induce the expression of hair inducing markers in DP cells. Therefore, it is important to use Wnt/ $\beta$-catenin signaling activators that activate both hair induction markers and stem cells to effectively promote hair growth and regeneration.

Although direct Wnt/ $\beta$-catenin signaling activators such as valproic acid promote hair growth, but they fail to sustain hair growth and often show marginal effects in clinical tests (Jo et al., 2013; Jo et al., 2014). This marginal and limited effect may be attributed to functions of negative feedback regulators such as CXXC5 or DKK1 (Lee et al., 2017; Kwack et al., 2012). We found that CXXC5, a negative feedback regulator of Wnt/ $\beta$-catenin signaling, is specifically increased in the miniaturized follicles of bald scalps, and $C X X C 5$ knock out mice exhibited enhanced hair growth (Lee et al., 2017). PTD-DBM, a peptide that interfered with CXXC5-Dvl protein interaction, enhanced hair growth, and the combinatory treatment of PTD-DBM and valproic acid synergistically increased hair growth and the WIHN (Lee et al., 2017). Similarly, KY19382, which strongly activated the Wnt/ $\beta$-catenin signaling via interference of the CXXC5-Dvl interaction and inhibited GSK-3 $\beta$ (Choi et al., 2019), critically enhanced hair re-growth as well as WIHNin vivo . The increased CXXC5 in bald scalps and the effectiveness of PTD-DBM or KY19382 on hair growth in mice suggest potential for use of KY19382 as hair growth treatment in the clinic.

In this study, we confirmed that the levels of $\beta$-catenin, p-GSK3 $\beta$ (S9) and proliferation marker, PCNA, were increased in human DP cells treated with KY19382. Increased ALP activity after KY19382 treatment suggested that KY19382 increased the hair inducing ability in human DP cells. Compared with the non-treated control, mouse vibrissa follicles treated with KY19382 for 6 days, significantly promoted elongation of hair shaft. The levels of $\beta$-catenin, Ki67, and PCNA were increased in the keratin 15-positive bulge of mouse skin treated with KY19382, suggesting a positive effect of KY19382 on hair follicle stem cell activity. Moreover, KY19382 regenerated a number of neogenic follicles in the WIHN assay, and histological images showed higher expression levels of not only $\beta$-catenin and proliferation markers but also markers for hair follicle neogenesis, fgf 9 and keratin 17. These results indicate that KY19382 treatment may be a possible therapy 
for baldness. In the hair patch assay, KY19382 regenerated a greater number of neogenic hair follicles, and histological evaluation revealed higher expression levels of $\beta$-catenin and Ki67 than control, demonstrating that pretreatment with KY19382 enhanced the hair-inducing ability of dermal cells. Therefore, our results suggest that KY19382 may be useful for treatment of hair loss and baldness via its effective dual targeting ability to inhibit both GSK-3 $\beta$ and CXXC5-Dvl interaction.

\section{ACKNOWLEDGEMENTS}

This work was supported by the National Research Foundation of Korea (NRF) grant funded by the Korean Government (MSIP) (2019R1A2C300275112). Y.C RYU was supported by a Brain Korea 21 (BK21) Plus studentship from the NRF.

\section{CONFLICT OF INTEREST}

K.Y.C is the CEO of CK Biotechnology Inc. (Seoul, Korea), which has a license to develop and use the compounds disclosed in the publication. The authors have no further conflicts of interest to declare.

\section{AUTHOR CONTRIBUTIONS}

Y.C.R., D.L., and J.P. performed and analyzed the experiments. Y.K., S.C., and S.S.B. helped in vitro and in vivo experiments. Y.C.R., J.S., S.L., and K.C. wrote the manuscript. Y.K.S., S.L., and K.C. supervised the study.

\section{ORCID}

Yeong Chan Ryuhttps://orcid.org/0000-0002-9270-5408

\section{REFERENCES}

Alonso, L., \& Fuchs, E. (2006). The hair cycle. J Cell Sci, 119 (Pt 3), 391-393. doi:10.1242/jcs02793

Andl, T., Reddy, S. T., Gaddapara, T., \& Millar, S. E. (2002). WNT signals are required for the initiation of hair follicle development.Dev Cell, 2 (5), 643-653.

Botchkarev, V. A., \& Kishimoto, J. (2003). Molecular control of epithelial-mesenchymal interactions during hair follicle cycling.J Investig Dermatol Symp Proc, 8 (1), 46-55. doi:10.1046/j.1523-1747.2003.12171.x

Choi, O. M., Cho, Y. H., Choi, S., Lee, S. H., Seo, S. H., Kim, H. Y., . . . Choi, K. Y. (2014). The small molecule indirubin-3'-oxime activates Wnt/beta-catenin signaling and inhibits adipocyte differentiation and obesity. Int J Obes (Lond), 38 (8), 1044-1052. doi:10.1038/ijo.2013.209

Choi, S., Kim, H. Y., Cha, P. H., Seo, S. H., Lee, C., Choi, Y., . . . Choi, K. Y. (2019). CXXC5 mediates growth plate senescence and is a target for enhancement of longitudinal bone growth. Life Sci Alliance, 2 (2). doi:10.26508/lsa.201800254

Enshell-Seijffers, D., Lindon, C., Kashiwagi, M., \& Morgan, B. A. (2010). beta-catenin activity in the dermal papilla regulates morphogenesis and regeneration of hair. Dev Cell, 18 (4), 633-642. doi:10.1016/j.devcel.2010.01.016

Han, J. H., Kwon, O. S., Chung, J. H., Cho, K. H., Eun, H. C., \& Kim, K. H. (2004). Effect of minoxidil on proliferation and apoptosis in dermal papilla cells of human hair follicle. J Dermatol Sci, 34 (2), 91-98. doi:10.1016/j.jdermsci.2004.01.002

Huelsken, J., Vogel, R., Erdmann, B., Cotsarelis, G., \& Birchmeier, W. (2001). beta-Catenin controls hair follicle morphogenesis and stem cell differentiation in the skin. Cell, 105 (4), 533-545.

Iida, M., Ihara, S., \& Matsuzaki, T. (2007). Hair cycle-dependent changes of alkaline phosphatase activity in the mesenchyme and epithelium in mouse vibrissal follicles. Dev Growth Differ, 49 (3), 185-195. doi:10.1111/j.1440-169X.2007.00907.x 
Ito, M., Yang, Z., Andl, T., Cui, C., Kim, N., Millar, S. E., \& Cotsarelis, G. (2007). Wnt-dependent de novo hair follicle regeneration in adult mouse skin after wounding. Nature, 447 (7142), 316-320. doi:10.1038/nature05766

Jang, W. S., Son, I. P., Yeo, I. K., Park, K. Y., Li, K., Kim, B. J., . . . Hong, C. K. (2013). The annual changes of clinical manifestation of androgenetic alopecia clinic in korean males and females: a outpatientbased study. Ann Dermatol, 25 (2), 181-188. doi:10.5021/ad.2013.25.2.181

Kishimoto, J., Burgeson, R. E., \& Morgan, B. A. (2000). Wnt signaling maintains the hair-inducing activity of the dermal papilla. Genes Dev, 14 (10), 1181-1185.

Kligman, A. M. (1959). The human hair cycle. J Invest Dermatol, 33 , 307-316.

Lee, E. Y., Choi, E. J., Kim, J. A., Hwang, Y. L., Kim, C. D., Lee, M. H., . . . Kang, S. (2016). Malva verticillata seed extracts upregulate the Wnt pathway in human dermal papilla cells. Int $J$ Cosmet Sci, 38 (2), 148-154. doi:10.1111/ics.12268

Lee, S. H., Seo, S. H., Lee, D. H., Pi, L. Q., Lee, W. S., \& Choi, K. Y. (2017). Targeting of CXXC5 by a Competing Peptide Stimulates Hair Regrowth and Wound-Induced Hair Neogenesis. J Invest Dermatol, 137 (11), 2260-2269. doi:10.1016/j.jid.2017.04.038

Lee, S. H., Yoon, J., Shin, S. H., Zahoor, M., Kim, H. J., Park, P. J., . . . Choi, K. Y. (2012). Valproic acid induces hair regeneration in murine model and activates alkaline phosphatase activity in human dermal papilla cells. PLoS One, 7 (4), e34152. doi:10.1371/journal.pone.0034152

Libecco, J. F., \& Bergfeld, W. F. (2004). Finasteride in the treatment of alopecia. Expert Opin Pharmacother, 5 (4), 933-940. doi:10.1517/14656566.5.4.933

Linas, S. L., \& Nies, A. S. (1981). Minoxidil. Ann Intern Med, 94 (1), 61-65.

Messenger, A. G., \& Rundegren, J. (2004). Minoxidil: mechanisms of action on hair growth. Br J Dermatol, $150(2), 186-194$.

Park, P. J., Moon, B. S., Lee, S. H., Kim, S. N., Kim, A. R., Kim, H. J., . . . Lee, T. R. (2012). Hair growth-promoting effect of Aconiti Ciliare Tuber extract mediated by the activation of Wnt/beta-catenin signaling. Life Sci, 91 (19-20), 935-943. doi:10.1016/j.lfs.2012.09.008

Paus, R. (2006). Therapeutic strategies for treating hair loss.Drug Discovery Today: Therapeutic Strategies, 3 (1), 101-110. doi:http://dx.doi.org/10.1016/j.ddstr.2006.03.004

Philpott, M. P., Green, M. R., \& Kealey, T. (1992). Rat hair follicle growth in vitro. Br J Dermatol, 127 (6), 600-607.

Price, V. H. (1999). Treatment of hair loss. $\quad N$ Engl J Med, 341 (13), 964-973. doi:10.1056/NEJM199909233411307

Rossi, A., Anzalone, A., Fortuna, M. C., Caro, G., Garelli, V., Pranteda, G., \& Carlesimo, M. (2016). Multitherapies in androgenetic alopecia: review and clinical experiences. Dermatol Ther . doi:10.1111/dth.12390

Sennett, R., \& Rendl, M. (2012). Mesenchymal-epithelial interactions during hair follicle morphogenesis and cycling. Semin Cell Dev Biol, 23 (8), 917-927. doi:10.1016/j.semcdb.2012.08.011

Sick, S., Reinker, S., Timmer, J., \& Schlake, T. (2006). WNT and DKK determine hair follicle spacing through a reaction-diffusion mechanism.Science, 314 (5804), 1447-1450. doi:10.1126/science.1130088

Waters, J. M., Richardson, G. D., \& Jahoda, C. A. (2007). Hair follicle stem cells. Semin Cell Dev Biol, 18 (2), 245-254. doi:10.1016/j.semcdb.2007.02.003

Xiao, Z., Hao, Y., Liu, B., \& Qian, L. (2002). Indirubin and meisoindigo in the treatment of chronic myelogenous leukemia in China.Leuk Lymphoma, 43 (9), 1763-1768. doi:10.1080/1042819021000006295 
Yamauchi, K., \& Kurosaka, A. (2009). Inhibition of glycogen synthase kinase-3 enhances the expression of alkaline phosphatase and insulin-like growth factor-1 in human primary dermal papilla cell culture and maintains mouse hair bulbs in organ culture. Arch Dermatol Res, 301 (5), 357-365. doi:10.1007/s00403009-0929-7

Zahoor, M., Cha, P. H., \& Choi, K. Y. (2014). Indirubin-3'-oxime, an activator of Wnt/beta-catenin signaling, enhances osteogenic commitment of ST2 cells and restores bone loss in high-fat diet-induced obese male mice. Bone, 65 , 60-68. doi:10.1016/j.bone.2014.05.003

Zahoor, M., Cha, P. H., Min do, S., \& Choi, K. Y. (2014). Indirubin-3'-oxime reverses bone loss in ovariectomized and hindlimb-unloaded mice via activation of the Wnt/beta-catenin signaling.J Bone Miner Res, 29 (5), 1196-1205. doi:10.1002/jbmr.2147

\section{Figure Legends}

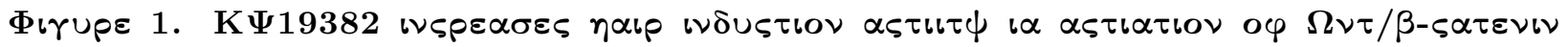
$\pi \alpha \tau \eta \omega \alpha \psi$. (a) TOPflash activity of HEK293T cells treated with the indicated concentrations of KY19382, I3O or DMSO for 24 hours $(\mathrm{n}=6)$. (b-e) Human DP cells were treated with the indicated concentrations of KY19382, $100 \mu \mathrm{M}$ MNX, or DMSO for 48 hours. (b) Cell viability of human DP cells (n=4). (c) Immunoblotting analysis for $\beta$-catenin, $\alpha$-tubulin, p-GSK3 $\beta$ and PCNA were analyzed using human DP cells. (d) Immunocytochemical staining for $\beta$-catenin. Nuclei were counterstained with DAPI (blue). Arrows indicate nuclei. $(\mathrm{n}=12)$ (e) ALP staining and (f) ALP activity test $(\mathrm{n}=3)$. (g) Transfected cells were subjected to ALP staining. (h) ALP activity was quantified $(\mathrm{n}=6)$. Scale bars $=100 \mu \mathrm{m}$. Values are expressed as the means \pm SEM. Student's t-test $\left(* \mathrm{P}<0.05,{ }^{* *} \mathrm{P}<0.01,{ }^{* * *} \mathrm{P}<0.001\right)$.

Figure 2. KY19382 accelerates mouse vibrissa follicle elongation. Mouse vibrissa follicles were cultured with $5 \mu \mathrm{M}$ KY19382 or $100 \mu \mathrm{M}$ MNX for 6 days. (a) The length of vibrissa follicles was measured at 6 days after treatment with $5 \mu \mathrm{M}$ KY19382 or $100 \mu \mathrm{M}$ MNX. Elongation rate of mouse vibrissa follicles was calculated as the difference in the length of vibrissa follicles wherein the vibrissa follicle length in the control group at day 6 was considered $100 \%$. Scale bars $=200 \mu \mathrm{m}$. (b) IHC analysis of mouse vibrissa follicle hair bulb for $\beta$-catenin and Ki67. Dashed lines mean mouse vibrissa follicles. (c) Mouse vibrissa follicles were subjected to ALP staining. Arrow indicates ALP-positive region. (d) X-gal staining of vibrissa follicles fromAxin $2^{l a c Z /+}$ mice. Arrow indicates X-gal-positive region. (b-d) Scale bars $=100 \mu \mathrm{m}$. Student's t-test $\left({ }^{*} \mathrm{P}<0.05,{ }^{* *} \mathrm{P}<0.01,{ }^{* * *} \mathrm{P}<0.001, \mathrm{n}=5\right)$.

Figure 3. KY19382 stimulates hair regrowth in vivo. Mice were treated with $0.5 \mathrm{mM}$ KY19382 or $100 \mathrm{mM}$ MNX for 14 or 28 days. (a) Gross image showing hair re-growth in mice treated with each drug for $28 \mathrm{~d}$ and quantitative measurements of weight of regenerated hairs. (b) $\mathrm{H} \& \mathrm{E}$ staining to evaluate the hair follicles of skins with different treatments and relative number of hair follicles and dermal thickness.

(c) IHC staining for keratin 15, $\beta$-catenin, and Ki67 using the dorsal skin of mice treated with each drug for 14 days. Lines show keratin 15-positive bulge stem cells region. (d) Immunoblotting analysis for $\beta$ catenin, PCNA, and ERK. (e) ALP staining was performed to evaluate the ALP activity of hair follicles with different treatments. Arrow points to ALP-positive region. Scale bars $=100 \mu \mathrm{m}$. Values are expressed as the means \pm SEM. Student's t-test $\left(* \mathrm{P}<0.05,{ }^{* *} \mathrm{P}<0.01,{ }^{* * *} \mathrm{P}<0.001, \mathrm{n}=5\right)$.

Figure 4. KY19382 stimulates WIHN in vivo. Mice wounds were treated with $0.5 \mathrm{mM}$ KY19382 or $100 \mathrm{mM}$ MNX for 14, 25, or 40 days. (a) ALP staining to evaluate the neogenic follicles of mice treated with each drug for 25 days and quantitative measurements of ALP-positive neogenic follicles. Dashed lines show ALP-positive neogenic hair follicles. (b) H\&E staining to evaluate newly formed hair follicles in mice treated with each drug for 14 days and the relative number of newly formed hair follicles. Dashed lines mean boundary between epidermis and dermis. Arrows show newly formed follicles (c) IHC staining for fgf 9, keratin 17, $\beta$-catenin, Ki67, and PCNA. (d) Immunoblotting analysis for fgf 9, keratin 17, $\beta$-catenin, PCNA, and ERK. (e) Gross images show newly formed hair in mice after treatment for 40 days. Scale bars $=100$ $\mu \mathrm{m}$. Values are expressed as means \pm SEM. Student's t-tests $\left(* \mathrm{P}<0.05,{ }^{* *} \mathrm{P}<0.01,{ }^{* * *} \mathrm{P}<0.001, \mathrm{n}=6\right)$. 
Figure 5. KY19382 induces hair follicle neogenesis in hairless mice. Mouse dermal cells were pretreated with $5 \mu \mathrm{M}$ KY19382 for 72 hours and then injected into hairless mice. The hair follicle neogenesis was analyzed on at 14 days after transplantation. (a) Hair follicle neogenesis at injected area of hairless mice. Arrow indicates hair regeneration area on the skin. (b) Magnified image of area exhibiting hair follicle neogenesis and quantitative analysis of regenerated hair follicles. (c) IHC analysis for $\beta$-catenin (scale bars $=50 \mu \mathrm{m})$ and quantitative analysis. Dashed lines mean hair follicles. Values are expressed as means \pm SEM. Student's t-tests $\left(* \mathrm{P}<0.05,{ }^{* *} \mathrm{P}<0.01,{ }^{* * *} \mathrm{P}<0.001\right)$.

\section{Supplementary Material}

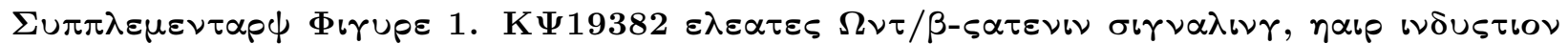

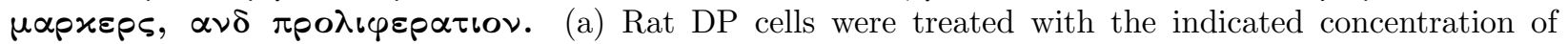
KY19382 for 48 hours. Cell viability was measured in rat DP cells that were treated with the indicated concentrations of KY19382 in 24 wells. (b) Rat DP cells were treated with the indicated concentrations of KY19382 or DMSO for 48 hours. Immunoblotting was performed to detect $\beta$-catenin and PCNA. (c) Immunocytochemical analysis for $\beta$-catenin (green) of rat DP cells. (d) Rat DP cells treated with 1 or $5 \mu \mathrm{M}$ of KY19382 were subjected to ALP staining via incubation with BCIP/NBT. Arrow indicated ALP-positive rat DP cells. (e) The ALP activity was quantified and normalized with respect to total protein concentration. Scale bars $=50 \mu \mathrm{m}$. Values are expressed as means \pm SEM. Student's t-tests $\left({ }^{*} \mathrm{P}<0.05,{ }^{* *} \mathrm{P}<0.01\right.$, ${ }^{* * *}$ $\mathrm{P}<0.001, \mathrm{n}=6)$.

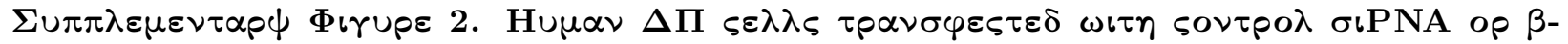

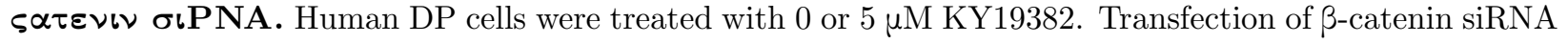
was confirmed through immunoblotting.

Supplementary Figure 3. KY19382 accelerates rat vibrissa follicle elongation. Rat vibrissa follicles from Wistar were cultured with 1 or $5 \mu \mathrm{M}$ KY19382 in DMEM for 12 days. (a) Elongation rate of rat vibrissa follicles was calculated as the difference in the length of vibrissa follicles wherein vibrissa follicle length in the control group at day 12 was considered $100 \%(n=8)$. (b) IHC analysis of rat vibrissa follicle hair bulb for $\beta$-catenin (green). Dashed lines were rat vibrissa follicles. (c) IHC analysis of rat vibrissa follicle bulge for $\beta$-catenin. Arrows indicate $\beta$-catenin positive nuclei. Scale bars $=50 \mu \mathrm{m}$. Student's t-tests (* $\left.\mathrm{P}<0.05,{ }^{* *} \mathrm{P}<0.01,{ }^{* * *} \mathrm{P}<0.001\right)$.

Supplementary Figure 4. Treatment with KY19382 increases proliferation marker in the dorsal skins of mice. Mouse dorsal skins were treated for 14 days. IHC staining for PCNA. Scale bars $=100 \mu \mathrm{m}$.

Supplementary Figure 5. Treatment with KY19382 enhances proliferation marker in mice wounds. Mice wounds were treated for 14 days. IHC analysis for PCNA. Scale bars $=100 \mu \mathrm{m}$. 
a

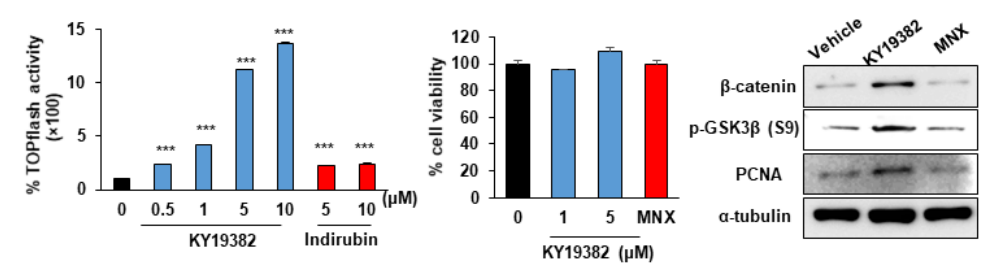

d Vehicle
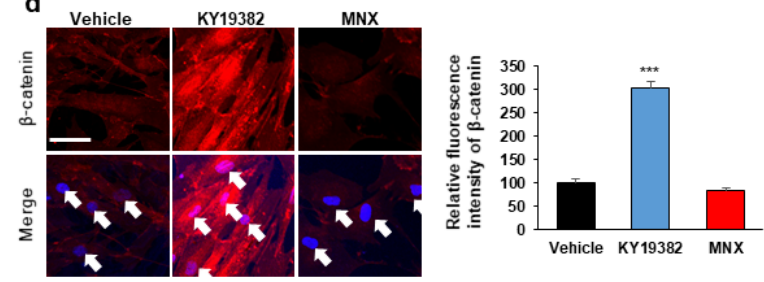

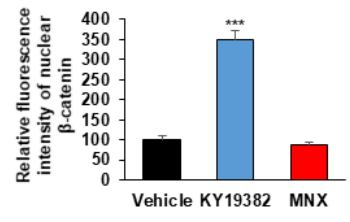

f

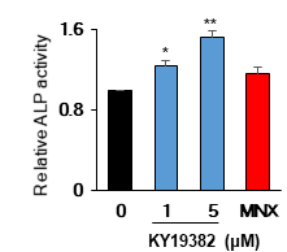

h

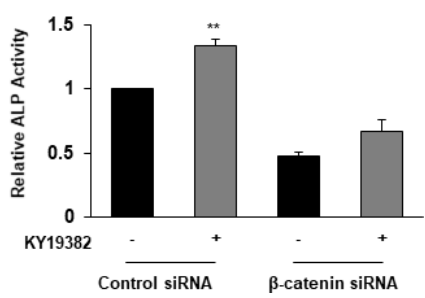



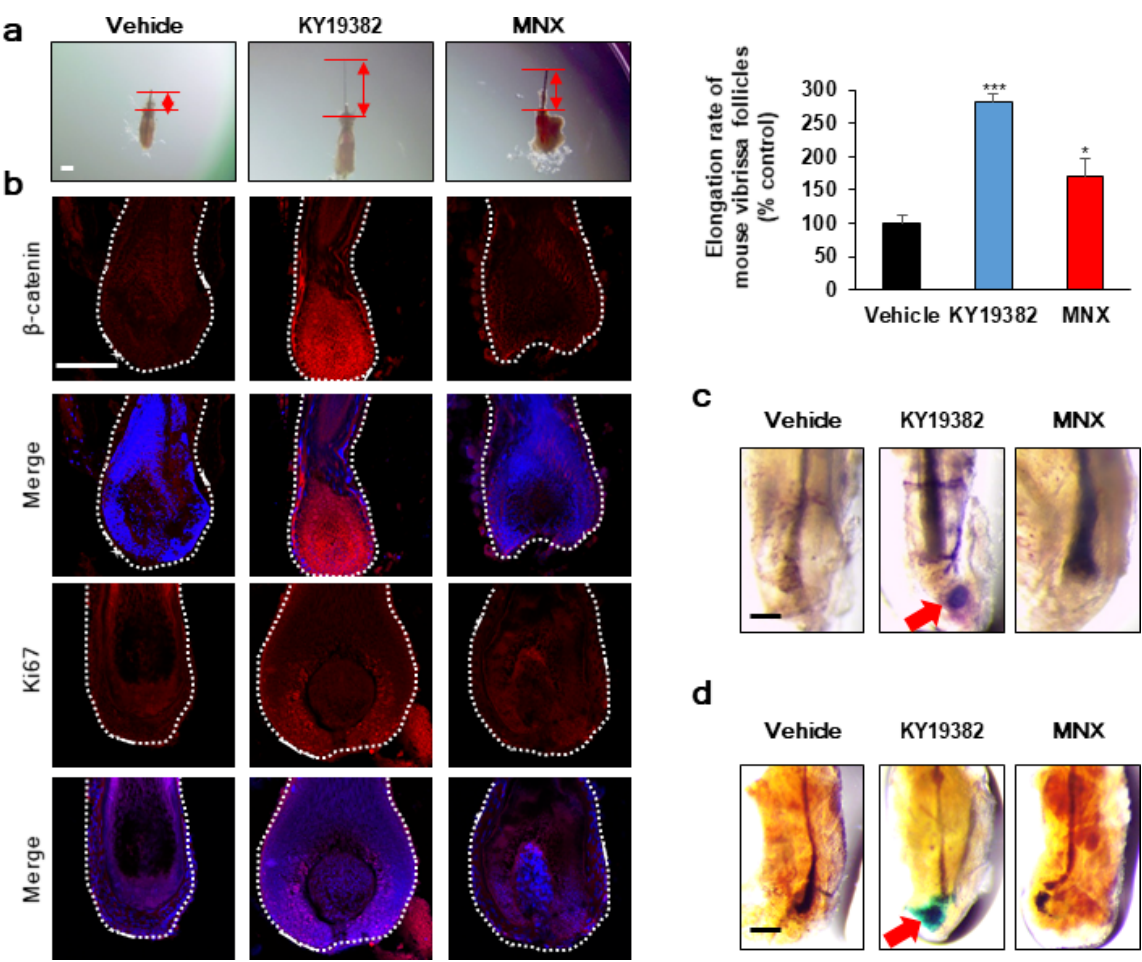

d
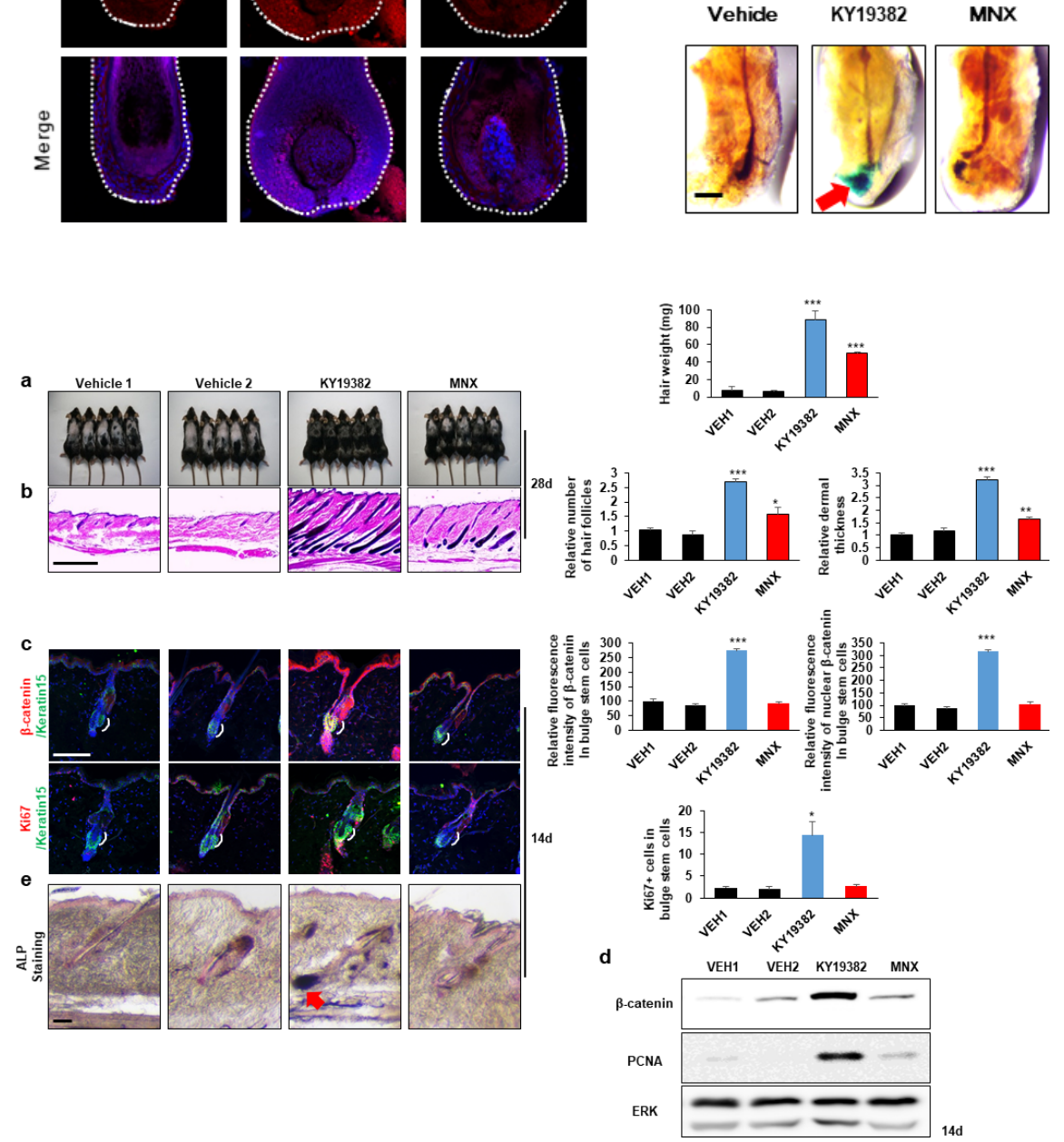

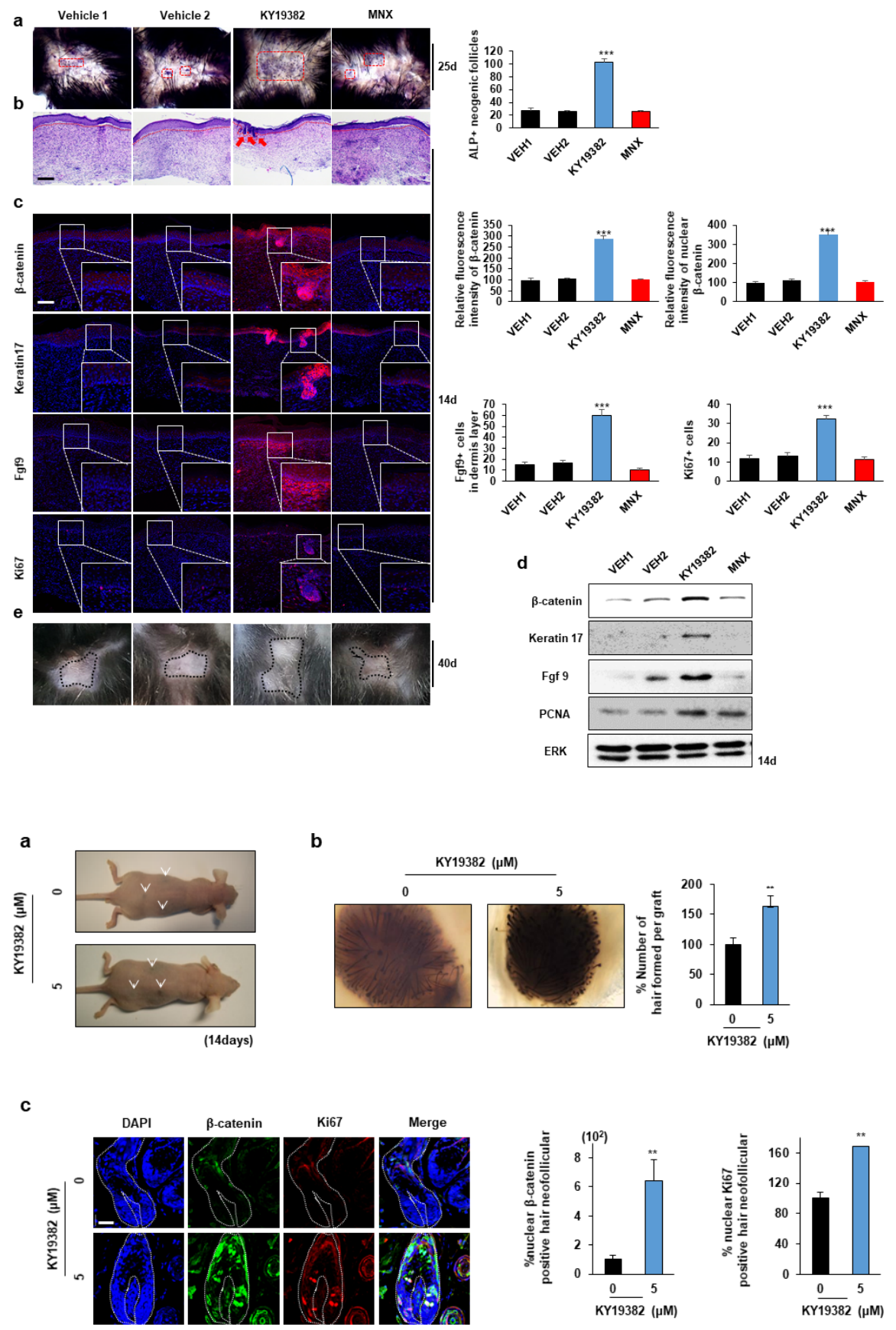